\title{
Geographical Variation in Multiple Lectins of the Acorn Barnacle Megabalanus rosa
}

\author{
Koji Muramoto, ${ }^{* 1}$ Ryusuke Kado,*1 Yoshiaki Yasui,*2 \\ and Hisao Kamiya*1 \\ (Received February 17, 1992)
}

\begin{abstract}
The multiple lectins in the acorn barnacle Megabalanus rosa collected from Sukumo Bay, Kochi, and Okirai Bay, Iwate, were analyzed for seasonal dynamics, agglutinating activities against animal erythrocytes, and peptide maps. Although there was no recognizable morphological difference between specimens collected from the two localities, heterogeneity in amino acid sequence was observed for the multiple lectins isolated from the barnacles. The present study shows that there is intraspecies genetic variations within $M$. rosa populations distributed along the Japanese coast.
\end{abstract}

Lectins are a group of sugar-binding proteins which recognize specific carbohydrate structures and agglutinate a variety of animal cells by binding to cell-surface glycoproteins and glycolipids. These properties make lectins useful biochemical reagents in many research areas such as separation and characterization of glycoproteins, and histochemistry of cells and tissues. In addition, the occurrence of lectins in different sub-species, species, or genera of the same family make them suitable markers for taxonomic and phylogenetic studies, as well as for evolutionary correlations. ${ }^{1}$

We have isolated multiple galactose-binding lectins from the coelomic fluid of the acorn barnacle Megabalanus rosa collected from Okirai Bay, Iwate.2) They are composed of three different molecular species with respect to molecular weight, designated oBRA-1 (Mr $330 \mathrm{kDa}$ ), oBRA$2(\mathrm{Mr} 140 \mathrm{kDa})$, and oBRA-3 (Mr $64 \mathrm{kDa})$. The lectins agglutinate rabbit and human erythrocytes and also several murine tumor cells, and induce binding of tumor cells to macrophages. oBRAs may be good models for the study of the biochemical and physiological functions of animal lectins, since their biochemical properies including the amino acid sequences have been well known. ${ }^{3-5)}$ Furthermore, the contents of multiple lectins in the coelomic fluid are high (approx. $20-30 \%$ of total proteins). Although $M$. rosa is one of the major fouling organisms and is distributed widely along the Japanese coast, from southern Sakhalin southward to the Yaeyama
Islands, ${ }^{6)}$ there is no information on intraspecies genetic variation within the population of $M$. rosa.

In this study, we describe the characterization of multiple lectins isolated from $M$. rosa collected from Sukumo Bay, Kochi, with special reference to the geographical variation in multiple lectins.

\section{Materials and Methods}

\section{Isolation and Quantitation of Lectins}

Galactose-binding lectins BRA-1, BRA-2, and BRA-3 were isolated from the coelomic fluid of the acorn barnacle $M$. rosa, as previously described." The acorn barnacles were collected from Okirai Bay, Iwate, and Sukumo Bay, Kochi. A lectin, MVA-1 (Mr $116 \mathrm{kDa}$ ), was also isolated from the coelomic fluid of $M$. volcano as described. ${ }^{7)}$

To examine the seasonal changes in the multiple lectin compositions of $M$. rosa in Sukumo Bay, barnacle specimens were collected monthly from May 1987 to April 1988. The shells of barnacles were broken with cutting pliers and the coelomic fluid was withdrawn using a pipet. The coelomic fluid $(300 \mathrm{~m} l)$ collected from 100 to 200 barnacles was centrifuged at $4^{\circ} \mathrm{C}$ for $20 \mathrm{~min}$ at $12,000 \mathrm{~g}$. The supernatant was applied to a column of acid-treated Sepharose 4 B $(2.5 \times 40 \mathrm{~cm})$ equilibrated with $50 \mathrm{~mm}$ Tris- $\mathrm{HCl}(\mathrm{pH} \mathrm{7.5)}$ containing $3.5 \%$ $\mathrm{NaCl}$ and $5 \mathrm{mM} \mathrm{CaCl}{ }_{2}$, and eluted with the same buffer. The lectins retained on the column were

*1 School of Fisheries Sciences, Kitasato University, Okirai, Sanriku, Kesen, Iwate 022-01, Japan (枯本光 二, 拀戸隆介, 神谷久男: 北里大学水尿学部).

*2 Cosmo Research Institute, Shibaura, Minato, Tokyo 105, Japan (安井義昆：コスモ総合研究所). 
eluted with $0.2 \mathrm{M}$ D-galactose in the buffer, dialyzed against several changes of deionized water for 2 days at $4^{\circ} \mathrm{C}$, and lyophilized. The lyophilized sample was dissolved in $50 \mathrm{~mm} \mathrm{NH}_{4} \mathrm{HCO}_{3}(\mathrm{pH}$ 8.0 ) at $10 \mathrm{mg} / \mathrm{m} /$ and subjected to high-speed gel filtration on a TSK G-3000 SW column $(2.15 \times$ $60 \mathrm{~cm}$, Tosoh) eluted with $0.25 \mathrm{M}$ sodium phosphate buffer $(\mathrm{pH} \mathrm{7.0)}$ at a flow rate of $2.0 \mathrm{~m} / /$ min. Eluates were monitored by uv absorption at $280 \mathrm{~nm}$. Each lectin quantity was determined from the peak area by calibration curves for purified lectins.

\section{Hemagglutinating Activity}

Estimation of agglutinating activity against various animal erythrocytes was carried out in multiwell microtiter plates using $2 \%$ erythrocyte suspension in $0.85 \% \mathrm{NaCl}$ containing $10 \mathrm{~mm}$ $\mathrm{CaCl}_{2}$. The activity was expressed by the minimum active concentration $(\mu \mathrm{g} / \mathrm{m} l)$ required for positive agglutination.

\section{Peptide Map}

Protein samples were reduced and alkylated with iodoacetamide as described. ${ }^{4}$ The modified proteins $(1 \mathrm{mg})$ in $1 \mathrm{ml}$ of $50 \mathrm{~mm} \mathrm{NH}_{4} \mathrm{HCO}_{3}$ were digested with TPCK-trypsin (Worthington) (200: $1, w / w)$ at $37^{\circ} \mathrm{C}$ for $4 \mathrm{~h}$. The digests were subjected to reversed-phase high-performance liquid chromatography (HPLC) on TSK ODS $120 \mathrm{~T}(4.6 \times 250 \mathrm{~mm})$. The column was eluted by a $60 \mathrm{~min}$ linear gradient from 0 to $60 \%$ acetonitrile in $0.1 \%$ trifluoroacetic acid (TFA).

\section{Analytical Methods}

Amino-acid analysis, sugar-analysis, and aminoacid sequence analysis were performed as described. 4 ,5)

\section{Results and Discussion}

We have isolated and characterized multiple galactose binding lectins from the coelomic fluid of the acorn barnacle, $M$. rosa which were collected from Okirai Bay. ${ }^{2)}$ They are composed of three different molecular species with respect to molecular weight, designated as oBRA-1 (Mr $330 \mathrm{kDa})$, oBRA-2 (Mr $140 \mathrm{kDa})$, and oBRA-3 (Mr $64 \mathrm{kDa}$ ). The coexistence of oBRAs in an individual was confirmed by SDS-gel electrophoresis followed by immunoblotting of its coelomic fluid, as well as by immunohistochemical study. ${ }^{8)}$ oBRA-3 is composed of four identical subunits ( $\mathrm{Mr} 16 \mathrm{kDa}$ ) of 138 amino acids. ${ }^{32}$ Both oBRA-1 and oBRA-2 are composed of identical subunits (Mr $22 \mathrm{kDa}$ ) which are a glycoprotein of 173 amino acids., oBRA-2 and oBRA-3 show $25 \%$ identity in the

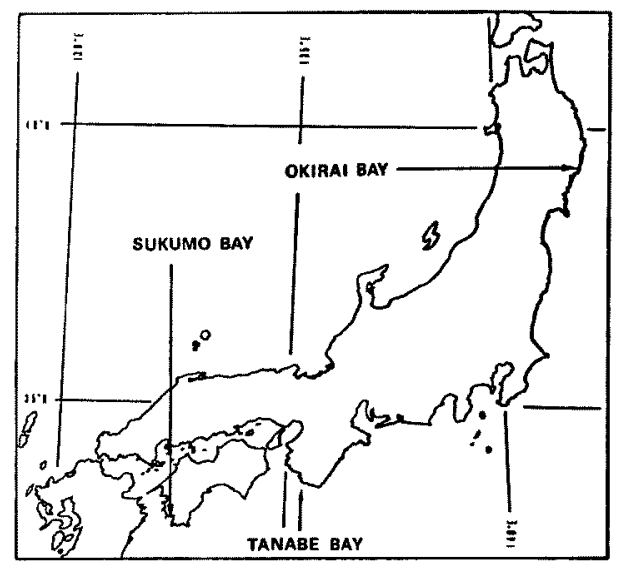

Fig. 1. Sampling localities.

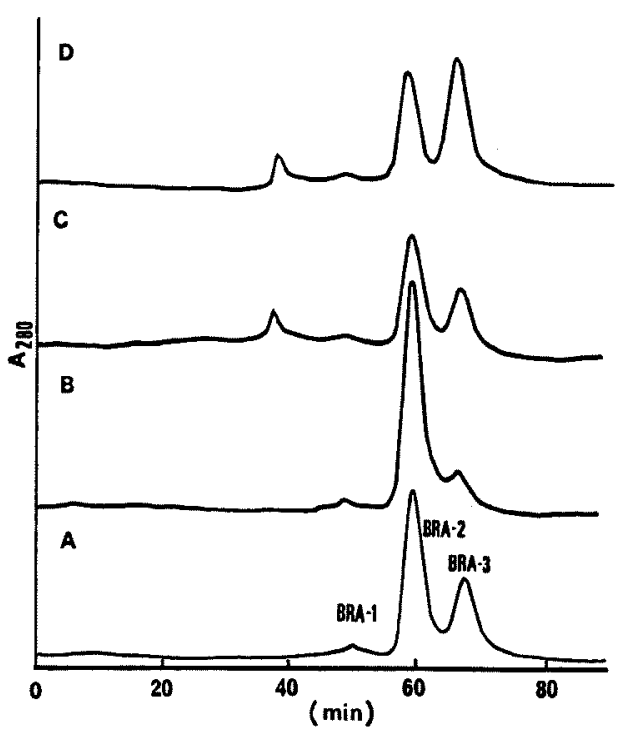

Fig. 2. Gel filtration on TSK G-3000 SW of multiple lectins in the coelomic fluid of $M$. rosa. The multiple lectins were isolated from the coelomic fluid collected in 1987 by affinity chromatography on HCl-treated Sepharose 4B. The lectins were dissolved in $50 \mathrm{~mm} \mathrm{NH}_{4} \mathrm{HCO}_{3}(10 \mathrm{mg} / \mathrm{ml})$ and injected into gel filtration. The column $(2.15 \times 60 \mathrm{~cm}$, Toso $)$ was eluted with $0.25 \mathrm{M}$ sodium phosphate $(\mathrm{pH} 7.0)$ at $2.0 \mathrm{ml} / \mathrm{min}$. A, $M$. rosa collected from Okirai Bay in May; B-D, $M$. rosa collected from Sukumo Bay in May, June, and July, respectively. 


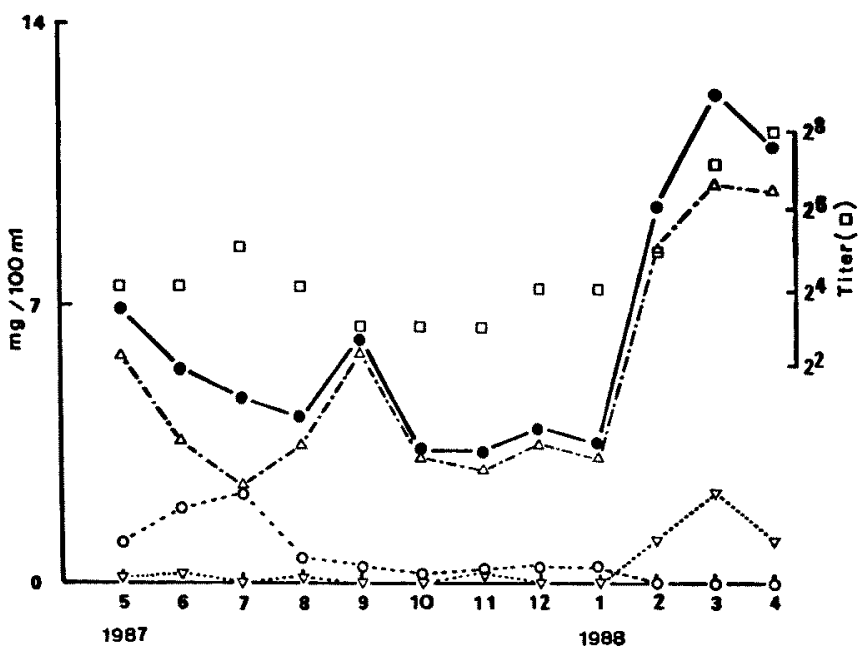

Fig. 3. Seasonal changes in multiple lectin compositions in the coelomic fluid of $M$. rosa. (•), Total amount; $(\nabla)$, BRA-1; $\triangle$, BRA-2; $(\bigcirc)$, BRA-3; $\square)$, agglutinating activity against rabbit erythrocytes.

Table 1. Minimum active concentration of $M$. rosa multiple lectins

$(\mu \mathrm{g} / \mathrm{m} l)$

\begin{tabular}{ccccccccc}
\hline Erythrocytes & Rabbit & Human A & Human B & Human AB & Human O & Sheep & Chicken & Horse \\
\hline sBRA-1 & 3.1 & 6.3 & 12.5 & 6.3 & 12.5 & $>50$ & 25.0 & $>50$ \\
SBRA-2 & 0.8 & 0.8 & 0.8 & 0.8 & 1.6 & 12.5 & 3.1 & $>50$ \\
sBRA-3 & 3.1 & 6.3 & 6.3 & 12.5 & 12.5 & $>50$ & 25.0 & $>50$ \\
oBRA-1 & 12.5 & 12.5 & 12.5 & 12.5 & 12.5 & $>50$ & $>50$ & $>50$ \\
oBRA-2 & 3.1 & 0.4 & 0.8 & 3.1 & 1.6 & $>50$ & 25.0 & $>50$ \\
oBRA-3 & 3.1 & 6.3 & 12.5 & 25.0 & 25.0 & 25.0 & $>50$ & $>50$ \\
\hline
\end{tabular}

sBRA: Lectins isolated from $M$. rosa collected from Sukumo Bay;

oBRA: lectins isolated from $M$. rosa collected from Okirai Bay.

amino acid sequence. In this study, specimens of $M$. rosa were collected monthly from Sukumo Bay, Kochi (Fig. 1). Galactose binding lectins were isolated from the coelomic fluid by affinity chromatography on HCl-treated Sepharose 4 B. They were separated into three fractions, designated as sBRA-1, sBRA-2, and sBRA-3, on a TSK G-3000 SW column as observed with $M$. rosa collected from Okirai Bay (Fig. 2).

The seasonal dynamics of multiple lectin compositions were analyzed by high-speed gel filtration chromatography under the same conditions described for Okiral samples. ${ }^{8)}$ The data obtained from the samples collected over the period from May 1987 to April 1988 are summarized in Fig. 3. The total amounts of multiple lectins varied from 3 to $12 \mathrm{mg} / 100 \mathrm{ml}$. A maximum level was observed in March 1988, though sBRA-3 was not detected. In early summer, the sBRA-3 level increased and the sBRA-2 level decreased, and they reached the same level in July. The seasonal dynamics of multiple lectins in the coelomic fluid of Sukumo samples were very similar to those of Okirai samples in spite of the shift of a time lag. This shift might be due to different reproductive cycles caused by environmental factors such as water temperature and food supply. We showed the seasonal dynamics of multiple lectins as related to the reproductive cycle of $M$. rosa collected from Okirai Bay. ${ }^{8}$ Annual cycles for total lectins show maximum levels in summer, and ovaries matured after that period. On the other hand, Yamaguchi ${ }^{\beta}$ reported that the breeding season of $M$. rosa was spring, when the water temperature began to rise in Tanabe Bay, Wakayama. Consequently, it is reasonable that Sukumo samples showed a maximum level of lectins in March, taking into account the localities. It should be noted that the total amounts of multiple lectins obtained from 
Table 2. Amino acid compositions of BRA-2 and BRA-3

\begin{tabular}{lcccc} 
& sBRA-2 & oBRA-2 & sBRA-3 & oBRA-3 \\
\hline Asp & 22.8 & 23 & 21.0 & 28 \\
Glu & 23.1 & 21 & 16.3 & 14 \\
Cys & 5.5 & 8 & 4.7 & 8 \\
Ser & 10.7 & 13 & 12.4 & 7 \\
Thr & 9.7 & 8 & 8.0 & 9 \\
Gly & 6.7 & 6 & 9.7 & 70 \\
Ala & 13.6 & 16 & 7.9 & 6 \\
Pro & 8.5 & 9 & 5.7 & 7 \\
Val & 12.9 & 15 & 6.6 & 2.5 \\
Arg & 9.8 & 7 & 2.6 & 1 \\
Met & 2.5 & 2 & 1.4 & 7 \\
Ile & 5.0 & 4 & 5.9 & 8 \\
Leu & 10.8 & 11 & 7.5 & 8 \\
Trp & 6.0 & 6 & 4.8 & 3 \\
Phe & 4.4 & 4 & 3.5 & 2.5 \\
Lys & 6.9 & 5 & 3.6 & 3 \\
His & 6.8 & 9 & 3.2 & 7 \\
Tyr & 6.9 & 6 & 6.8 & 7 \\
\hline
\end{tabular}

SBRA, Lectins isolated from $M$. rosa collected from Sukumo Bay:

oBRA, lectins isolated from $M$. rosa collected from Okiral Bay.

Values for oBRA-2 and oBRA-3 are from the amino acid sequences, 3,4 )
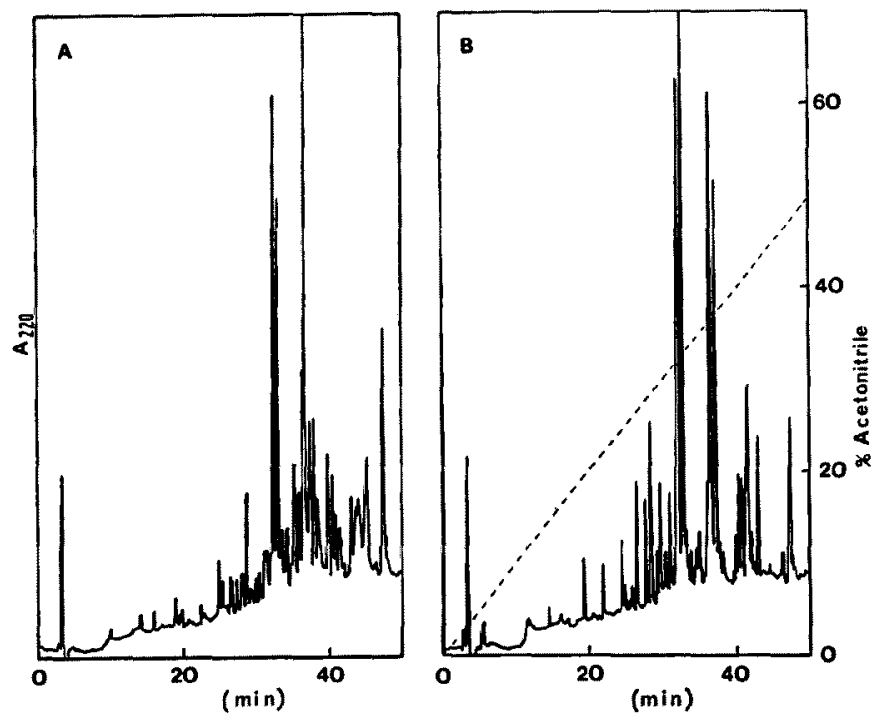

Fig. 4. Peptide maps of CAM-BRA-3. Tryptic digests of CAM-BRA-3 $(0.1 \mathrm{mg})$ were subjected to reversed-phase HPLC on TSK ODS $120 \mathrm{~T}(5 \mu \mathrm{m}, 4.6 \times 250 \mathrm{~mm})$. The separation was achieved using a linear gradient of acetonitrile in $0.1 \%$ TFA at a flow rate of $1.0 \mathrm{~m} / \mathrm{min}$. A, CAMSBRA-3; B, CAM-oBRA-3.

Sukumo samples $(3-12 \mathrm{mg} / \mathrm{m} l)$ were smaller than those from Okirai samples $(14-52 \mathrm{mg} / \mathrm{ml})$. We have no explanation for this observation.

The results of agglutinating reaction of sBRAs and oBRAs with various animal erythrocytes are summarized in Table 1, sBRAs and oBRAs agglutinated rabbit and human erythrocytes at concentrations from 0.4 to $25 \mu \mathrm{g} / \mathrm{m} /$. sBRAs showed higher agglutinating activity than oBRAs against human AB. Chicken erythrocytes were 

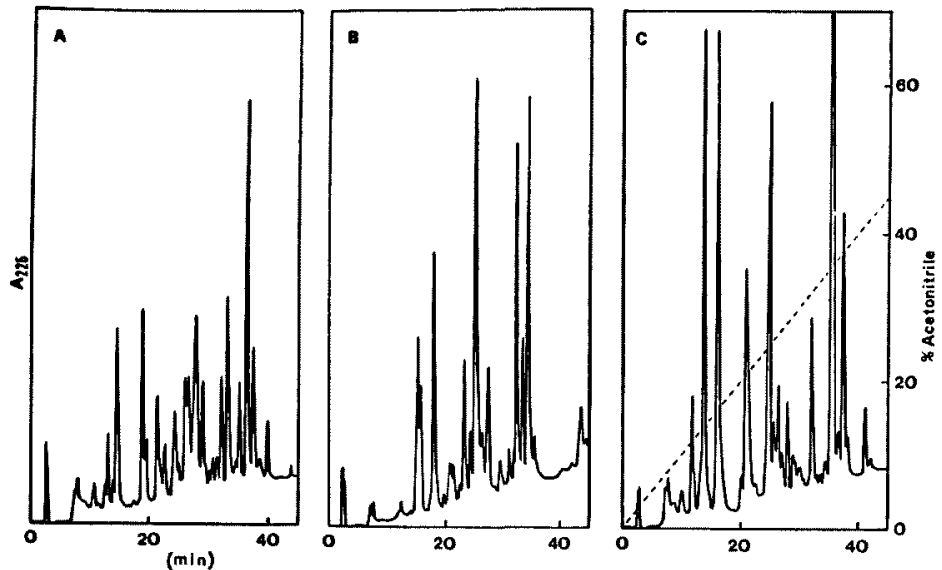

Fig. 5. Peptide maps of CAM-BRA-2 and CAM-MVA-1. Tryptic digests of CAM-BRA-2 and CAM-MVA-1 (1 mg) were subjected to reversed-phase HPLC on TSK ODS $120 \mathrm{~T}(10 \mu \mathrm{m}$, $4.6 \times 250 \mathrm{~mm}$ ). The separation was achieved using a linear gradient of acetonitrile in $0.1 \%$ TFA at a flow rate of $1.0 \mathrm{~m} / \mathrm{min}$. A, CAM-sBRA-2; B, CAM-oBRA-2; C, CAM-MVA-1.

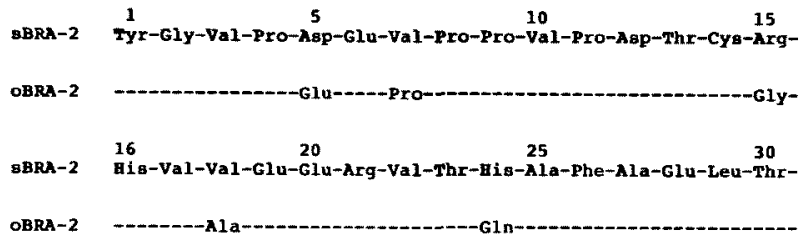

Fig. 6. A comparison of the amino-terminal amino acid sequence of sBRA-2 with that of oBRA-

2. Residues homologous are indicated with a dashed line.

agglutinated with $S B R A s$ at concentrations from 3.1 to $25 \mu \mathrm{g} / \mathrm{m} l$. On the other hand, only oBRA2 among oBRAs showed weak agglutinating activity against chicken erythrocytes. None of the BRAs agglutinated horse erythrocytes at the concentrations examined.

The amino acid compositions of SBRA-2 and SBRA-3 were compared with those of oBRA-2 and oBRA-3 (Table 2). Although sBRA-2 and SBRA-3 showed similar amino acid compositions to each corresponding oBRA, some significant differences were observed with the values of a few amino acids, such as Ala, Val, Arg, and His for BRA-2, and Asp, Ser, and Trp for BRA-3. oBRA-2 is a glycoprotein which contains $0.6 \%$ mannose, $0.2 \%$ fucose, and $2.3 \% \mathrm{~N}$-acetylglucosamine by weight. SBRA-2 showed the same sugar compositions.

The amino acid sequences of sBRAs were compared with those of oBRAs by peptide mapping using HPLC. BRAs were reduced and alkylated with monoiodoacetamide, digested with trypsin, and analyzed using reversed-phase HPLC. $S$ Carboxamidomethylated (CAM-) sBRA-3 and CAM-oBRA-3 showed similar peptide maps, though not the same, indicating that there is a significant sequence homology between sBRA-3 and oBRA-3 (Fig. 4). On the other hand, CAMSBRA-2 and CAM-oBRA-2 gave quite different elution profiles, indicating that they have distinct amino acid sequences (Fig. 5).

CAM-sBRAs were then applied to a gasphase sequencer (Shimadzu PSQ-1) to analyze amino-terminal amino acid sequence. The first 30 amino-terminal amino acid residues of sBRA-2 were identified and compared with the sequence of oBRA-2 (Fig. 6). Amino acid residue alterations between SBRA-2 and oBRA-2 were found in positions $5,7,15,18$, and 24 . Thus far we have analyzed about $60 \%$ of the amino acid sequence of sBRA-2, showing $25 \%$ sequence discrepancy between sBRA-2 and oBRA-2 (data not shown). No sequence heterogeneity among sBRA-2 or oBRA-2 samples, which were isolated from $M$. 
rosa collected in different seasons, has been detected. The amino acid sequence of a putative carbohydrate binding site corresponding to positions 106-112 was common between them. With SBRA-3, no replacement was detected in the amino acid sequence up to 30th residues from the amino-terminus. As a result, $M$. rosa collected from two localities have the distinct genes coding BRA-2, thus, there is intraspecies genetic variation within the species distributed along the Japanese coast. The various agglutinating activities among the lectins from the two localities are probably attributed to the genetically separate races. Racial differences in a barnacle species is substantiated in Semibalanus balanoides by the heterogeneity of several esterase activities. ${ }^{\text {g) }}$ The species has wide range of distribution, and is very different from $M$. rosa with respect to its rather local distribution. Therefore BRA-2 could be a suitable clue for the separation of races it the present variation were due to different races, even in local species.

$M$. volcano is phylogenetically related to $M$. rosa and their distribution overlaps in Kochi. Yamaguchi ${ }^{(0)}$ distinguished between $M$, rosa and $M$. volcano as different species by morphological characters, isozymes, and several factors of field evidence such as differentiation of breeding seasons. Nevertheless, a lectin, MVA-1, isolated from $M$. volcano is similar in subunit structure to BRA2 ; that is, MVA-1 is composed of a subunit with a molecular weight of 23,000 , which is crosslinked by disulfide bonds to form dimers. ${ }^{\text {") }}$ A peptide map of MVA-1 was compared with those of $M$. rosa (Fig. 5). No similarity among the peptide maps reconfirmed the taxonomic identification of Megabalanids made by Yamaguchi. ${ }^{0}{ }^{-}$

\section{Acknowledgments}

This work was supported in part by a Grantin-Aid from the Fisheries Agency, Japan, and a grant from the Research Institute of Marine Invertebrates.

\section{References}

1) N. Sharon and H. Lis: in "Lectins", Chapman and Hall, New York, 1989, pp. 1-114.

2) K. Muramoto, K. Ogata, and H. Kamiya: Comparison of the multiple agglutinins of the acorn barnacle, Megabalanus rosa. Agric. Biol. Chem., 49, 85-93 (1985).

3) K, Muramoto and $H$. Kamiya: The amino-acid sequence of a lectin of the acorn barnacle Megabalanus rosa. Biochim. Biophys. Acta, 874, 285-295 (1986).

4) K. Muramoto and H. Kamiya: The amino-acid sequence of multiple lectins of the acorn barnacle Megabalanus rosa and its homology with animal lectins. Biochim. Biophys. Acta, 1039, 42-51 (1990)

5) K. Muramoto and H. Kamiya: The positions of the disulfide bonds and the glycosylation site in a lectin of the acorn barnacle Megabalanus rosa. Biochim. Biophys. Acta, 1039, 52-60 (1990).

6) T. Yamaguchi: On Megabalanus (cirripedia, thoracica) of Japan. Publ. Seto Mar. Biol. Lab., 21, 115-140 (1973).

7) H. Kamiya, K. Muramoto, and R. Goto: Isolation and characterization of agglutinins from the hemolymph of an acorn barnacle, Megabalanus volcano. Develop. Comp. Immun., 11, 297-307 (1987).

8) K. Muramoto, R. Kado, Y. Takei, and H. Kamiya: Seasonal changes in the multiple lectin compositions of the acorn barnacle Megabalanus rosa as related to ovarian development. Comp. Biochem. Physiol, 98B, 603-607 (1991).

9) M. W. Flowerdew and D. J. Crisp: Esterase heterogeneity and an investigation into racial differences in the cirripede Balanus balanoides using acrylamide gel electrophoresis. Mar. Biol., 33, 33-39 (1975). 